\title{
Power Control Mechanism in OFDM Based Cognitive Radio System
}

\author{
Siyaram Yadav, Dharmendra Kumar \\ ECE Department, Mewar University, Chittorgarh, India
}

\begin{abstract}
Power is key parameter in communication system. In cognitive radio power of transmitter of primary user and secondary user should be at appropriate level for faithfull reception, at the same time there should be no interference between and among the adjacent users. In this paper power control mechanism is proposed for determining the primary user transmitter power. Interference caused due to primary user to secondary user is white noise by secondary user imply different noise levels in different sub channels. Here OFDM is used to fill the spectral gap for which OFDM channel filling optimization technique is used.
\end{abstract}

Keywords: Cognitive Radio,OFDM,Primary User, Secondary User,FFT.

\section{Introduction}

With the increase in wireless users the spectrumis becoming scarce.Present method of frequency allocation combined with a reliance on fixed infrastructure threatens to halt this growth. An additional consequence is the deployment of fundamentally less robust system, prone to disruption in major disaster or overload. By enabling the secondary use of spectrum on an opportunistic basis, ubiquitous, robust and agile wireless system can be realized that are able to support further traffic growth and changing demands in traffic, while ensuring operation in case of emergencies. As such, it will enable the extension of wireless data-rates and coverage's for many decades to come and open the door for exciting new application to emerge.

Searchfor the methods and techniques to utilize spectrum efficiently halts at implementation of CR [1].At the same time transmitted power could be reduced. In conventional communication systems due to static spectrum allocation it couldn't be used efficiently [2]. FCC recommendation empowers unlicensed users (secondary users) to licensed user's (primary user) band so that whenever primary user(PU) is not able to exploit the bandwidth available to them then secondary users (SU) may utilize the available bandwidth i.e. free channels called white spaces on the opportunity basis. Such a radio may be called opportunistic radio or cognitive radio. Cognitive radio is characterized by its dynamic spectrum sensing capability; low transmit power, less co-channel interference, good signal strength, good efficiency, flexible modulation technique, fast processing and its versatility.Further the communication may become economic at the cost of complexity.It is found that spectrum uses is not efficient [3]. Any optimization algorithm may be utilized some of them are Game theory, Temperature Interference Model, Genetic Algorithm, Power Control Mechanism Optimization, OFDM Channel Filling, Fuzzy Logic Optimization, Load Smoothing Algorithm, Recursive Power Allocation Algorithm, Iterative Partitioned Water Algorithm. If SNR level of PU falls below the threshold then reliable detection becomes difficult even if an indefinitely long observation is made available. Cognitive transmission in spectrally sparse and dense environment leads to less throughput. In cognitive transmission proper interference management is required [4]. If PU channel is not fully loaded it can tolerate cognitive user interference [5]. The cognitive transmission parameters design in accordance with the parameters of the primary users so that quality of service of PU is not affected. So we take quality of service measure as the MSE(Mean Square Estimation Error) of the PU signal in case of uncoded PU transmission and as the achievable information rate of encoding the PU message in case of coded PU transmission [6]. Power level of the interference temperature, power spectral density describes the achievable performance of the PU receiver. Optimizing the PSD of cognitive signal is called spectrum shaping. This can be done in two ways

(1) By encoding the message signal digitally.

(2) Without encoding

by the primary user. Spectrum sharing is also investigated in this paper. We found that, through appropriately shaping the cognitive signal spectrum coupled with optimal filtering (for the case of uncoded legacy transmission) or decoding (for the case of coded legacy transmission), cognitive transmission can actually overcome the interference limitation, and can always transmit at its full available device power. Cognitive transmission is forbidden to keep ramping its power level when hitting a certain upper limit which is much smaller than the available device in short distance case [7]. Logarithmic growth rate of cognitive transmission in high power resign is relevant and advantageous in spectrum shaping approach [8]. Logarithmic growth rate of cognitive transmission rate is always supported when primary channel is not fully loaded. 
Spectrum shaping approach leads to a subset of spectral bands that are turned on while leaving the remaining spectral bands off. This is analogous to FDMA and bear optimal properties in spectral sharing among multiple cognitive systems for unliscensed band [9]. Liscened band are occupied by PU so FDMA sharing method is not applicable. The on/off behavior of cognitive signal PSD is the more a consequence of non-convex optimization [10]. Dirty paper coding algorithm is strongly recommended one. In this cognitive transmitter has acces to the message of PU transmitter with this additional knowledge the cognitive transmitter can adapt its signal using dirty paper coding so as to compensate for the excess interference it causes to the legacy receiver and the overall effect is that the legacy transmission is not affected by cognitive transmission. This also supports logarithmic growth of cognitive transmission rate and does not reduce pre-log coefficient [11]. This leads to complexity and non-ideal effects in implementation.Scalability, privacy and security, legacy message sharing is unlikely feasible due to technological and political constraints. In case of uncoded legacy transmission the interference temperature model is one of the candidates.

\section{Ofdm Based System}

Orthogonal Frequency Division Multiplexing isspectrally efficient modulation technique because in its spectrum carrier overlaps and those carrier remain orthogonal to eachother which inceasesthe BER (SNR) to the signal carrier transmission [12]. OFDM with wavellet bases is utilized nowadays because of its flexibility and adaptability. This proves to be a very strong modulation technique that is to be utilized in cognitive radio environment. It helps cognitive radio to analyse the spectrum presently in use. FFT algorithm is used for spectral analysis.

FFT is also used as demodulator for OFDM. OFDM is capable of notching the part of its carrier which are co-incidentally within the region of PU's band [13]. This property is exploited in dynamic spectrum acces by the cognitive radio.Spectrum Pooling Technique is explained in subsequent section. Large side lobes that results in interference between subcarriers originating from different secondary users and between PU and SU. The side lobes of Fourier-based OFDM can be observed from its power spectral density

$$
P S D(f)=\frac{1}{N_{F F T}}\left|\sum_{m=0}^{N_{F F T-1}} S_{m} \sqrt{p_{m}} \int_{-(1+\alpha) \frac{T_{u}}{2}}^{(1+\alpha) \frac{T_{u}}{2}} g(t) e^{-j 2 \pi\left(f-f_{m}\right) t} d t\right|^{2}
$$

Where $N_{F F T}$ is the number of FFT points, $\mathrm{T}_{\mathrm{u}}$ isusefull signal duration, $\mathrm{g}(\mathrm{t})$ is window function, $\alpha$ is role of factor of the window, while

$\mathrm{f}_{\mathrm{m}}$ is frequency of the sub carrier $\mathrm{m}$

$\mathrm{P}_{\mathrm{m}}$ is allocated power of the sub carrier $\mathrm{mS}_{\mathrm{m}}$ is symbol amplitude from quadrature amplitude modulation (QAM) or phase shift keying (PSK) on the sub carrier $\mathrm{m}_{\mathrm{m}}, \mathrm{S}_{\mathrm{m}}, \mathrm{g}(\mathrm{t})$ resolves side lobe problem. The method of reduction of side lobe gives rise to lose in band width because excessive time allocation has to be made to cyclic extensions which could be used for data transmission. Sub carrier near to primary user is deactivated for less interference. For this RCT (Raised Cosine Technique) is used. The other available forms are Bartlett, better than raised cosine (BTRC), and flipped inverse hyperbolic secant (farchsech) window [14]. Of this window, it is the farchsech window that provides the lowest side lobes. The window is designed according to equation of $\mathrm{g}(\mathrm{t})$

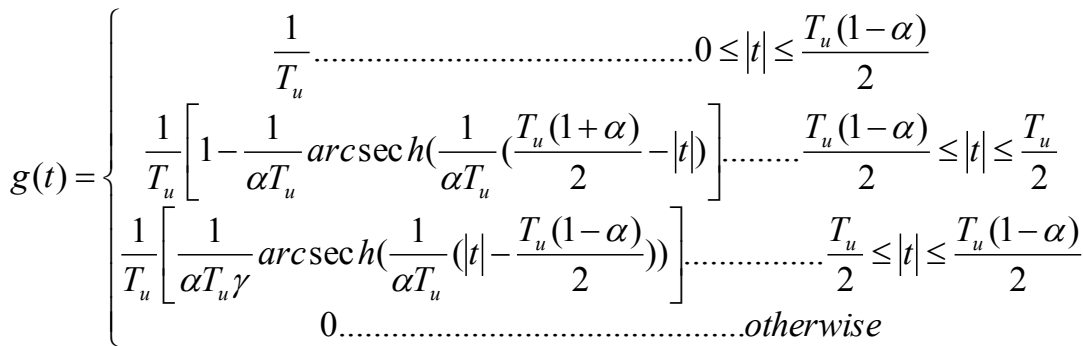

This zeros insertion impact can be described from TDCS

$$
P S D(f)=\left|\frac{1}{N_{F F T}} \sum_{m=0}^{N_{F F T-1}} A_{m} e^{-j 2 \theta_{m}} \int_{-(1+\alpha) \frac{T_{u}}{2}}^{(1+\alpha) \frac{T_{u}}{2}} p(t) e^{-j 2 \pi\left(f-f_{m}\right) t} d t\right|^{2}
$$


Due to the extra embedded symbol, the TDCS PSD would become

$$
P S D(f)=\left|\frac{1}{N_{F F T}} S e^{j \theta} \sum_{m=0}^{N_{F F T-1}} A_{m} e^{-j \theta_{m}} \int_{-(1+\alpha) \frac{T_{u}}{2}}^{(1+\alpha) \frac{T_{u}}{2}} p(t) e^{-j 2 \pi\left(f-f_{m}\right) t} d t\right|^{2}
$$

In multicarrier wavelet packet modulation (MC-WPN), the Fourier transform used in OFDM is replaced by the orthogonal wavelet packet bases. The wavelet packet reconstruction two-band finite-impulse response (FIR) filter bank solution using multistage tree-structured paraunitary filter banks derived by cascading two-channel filter banks. The wavelet transform provides higher sidelobes in regular OFDM. In the Daubechies and Haar wavelet-based multicarrier transmission over Rayleigh fading channel were evaluated. Their performances in terms of intersymbol interference (ISI) and intercarrier interference (ICI) have been compared with the Fourier- based OFDM using rectangular window. The wavelet-based multicarrier provides significant ICI power reduction and slight ISI power reduction compared to OFDM.

\section{Wavelet Packet Filterbank}

The wavelet packet subcarrier (to be used at the transmitter end) are generated through a multichannel filterbank in which the synthesis filter $(\mathrm{H}$ and $\mathrm{G})$ are applied. The process know as the synthesis procedure consists of binary interpolation (upsampling) by two, filtering and recombination at each level. To demonatrate the generation at each level. To demonstrate the generation of the wavelet packet bases process, we shall consider here a cascaded level-three structure of the type. Such an arrangement can give rise to eight wavelet packet bases. In general, a level-j tree has to be constructed to generate M bases or subcarrier waveforms. The structure can be simplified by applying the nobel identities procedure that will produce multirate systems. Each of the carrier will be upsampled by $\mathrm{M}$ and will experience only one tap filtering. The filter is the equivalent filter resulting from the convolution of the combination between low pass and high pass filter according to the branch line of the tree. The high pass filter H'(z) with length L' can be obtained from the low pass filter G'(z) by means of the $G^{\prime}(z)=-z^{-\left(L^{\prime}-1\right)} H^{\prime}\left(-z^{-1}\right)$ equation. The coeixtence with the LUs is actualized by shaping its transmission waveform and by adaptively activating or deactivating subcarriers in such a way as to utilize the unoccupied time-frequency gaps of the LU. The idea is to dynamically notch the transmission signal so that it has no or very little time-frequency components competing with the LU. In that way, the CR can seamlessly blend with the LU operation. The wavelet packet duals(to be used at the receiver end) are also obtained from multichannel filter bank analysis, though the processes are reserved. The duals are obtained from the analysis filters ( $\mathrm{H}$ and $\mathrm{G}$ ) through the analysis procedure which consist of filtering, decimation (downsampling) by two and decomposition at each stage. The dual filters are derived from paraunitarycondition $P(z)+P(-z)=2$, where the product filer $P(z)=H(z) H\left(z^{-1}\right)$ And the $H\left(z^{-1}\right)=H^{\prime}(z)$. In the time domain the analysis filters are the complex conjugate time reversed version of the synthesis filters $\left(h^{\prime}(n)=h^{*}(-n)\right.$ and $g^{\prime}(n)=g^{*}(-$ $\mathrm{n})$ ). The wavelet basis function in the application of MC-WPM system should fulfill some fundamental properties. These properties include the time and band limitation, the orthogonality between the bases and their duals for perfect reconstruction, and the orthogonality between the bases for the unique demodulation of each basis function [15].

\section{Conclusion}

In this paper power control mechanism for OFDM based cognitive radio have been investigated. OFDM is used to fill the spectral gap for which OFDM channel filling optimization technique is used.In this paper power control mechanism is proposed for determining the primary user transmitter power. Interference caused due to primary user to secondary user is white noise by secondary user imply different noise levels in different sub channels.In this chapter, we study the power allocation problem in OFDM-based cognitive radio systems. In the cognitive radio scenario, the interference limits of the primary users introduce subchannel power constraints for the transmission of the SU. Therefore, the power allocation among the subcarriers should satisfy both the sum transmit power constraint, as that in conventional OFDM systems, as well as the subchannel power constraints, which leads to the unavailability of the well-known water-filling algorithm applied in conventional OFDM systems.

In order to find the optimal power allocation, we first propose the IPW algorithms, whose basic computation units is the conventional water-filling. The algorithm is proved to converge to the optimal power allocation, which maximizes the sum rate when the sum transmit power constraint is given or minimizes the 
required power when the target rate is given, after a finite number of iterations. Furthermore, we consider the effects of subcarrier sidelobes on the power allocation in practical systems. We note that the actual transmit power in one subchannel is comprised of the allocated power as well as the subcarrier sidelobes power from the neighboring subchannels. The RPA algorithm is proposed for this scenario. The algorithm finds the optimal power allocation recursively by decoupling the subchannel power constraints phase-by-phase. Simulation results show that the IPW algorithm is unavailable when the guard bands between the subchannels are not wide enough, while the RPA algorithm can be applied in such scenario. In conventional OFDM systems, bit loading is a more practical technique for the system design. Therefore, it is interesting to study bit loading algorithms in OFDM-based cognitive radio systems, where the subchannel transmit power constraints should be considered.

\section{References:}

[1]. J. Mitola and G. Q. Maguire, "Cognitive radios: Making software radios more personal," IEEE Pers. Commun., vol. 6, no. 4, pp. 1318, Aug. 1999

[2]. J. Mitola, "Cognitive radio: An integrated agent architecture for software defined radio," Ph.D. dissertation, KTH, Royal Institute of Technology, Sweden, 2000.

[3]. Fette B "Cognitive radio Technology" ISBN-13: 978-0-7506-7952-7, Newnes 2006.

[4]. S. Haykin. Cognitive Radio: Brain-Empowered Wireless Communications. IEEE J. Sel. Areas Commun., 23(2):201-220, Feb. 2005.

[5]. MohamedGafar Ahmed Elnourani "CR and game theory: overview and simulations" Master's Thesis 2008

[6]. Bin Le "Building a cognitive radio: from architecture definition to prototype implementation" $\mathrm{PhD}$ thesis 2007

[7]. Chen K.C. "CR network architecture part 1 structure" 2007.

[8]. Ying-Chang Liang, YonghongZeng, Edward C.Y. Peh, and Anh Tuan Hoang, "Sensing throughtput tradeoff for CR networks" IEEE transactions on wireless communications, vol. 7, no. 4, april 2008

[9]. Proakis, John G. Digital Communications. McGraw-Hill College, 2000.

[10]. Lathi B.P. Principles of Digital and Analog communication Oxford University press.

[11]. Van Nee, Prasad R. OFDM for Wireless Multimedia Communications. Boston (USA): Artech House, 2000.

[12]. Thomas Charles Clancy III "dynamic spectrum access in cognitive radio networks". Ph.D Thesis 2006 pp 34

[13]. Akyildiz F, Lee, VuranMohantyS "NeXtgenerationdynamic spectrum acess cognitive radio wireless networks: A survey" 2006.

[14]. J. Acharya and R. D. Yates, "A framework for dynamic spectrum sharing between cognitive radios," in Proc. ofIEEE ICC 2007, Glasgow, 2007, pp. 5166-5172.

[15]. Wang P, Chen X, Zhong X, Xiao L “Power Allocation in OFDM Based Cognitive Radio systems" 2012. 